\title{
„Wenn wir nicht mehr wachsen, wie verteilen wir dann um?"6
}

\author{
Die Rolle von Gewerkschaften bei der Gestaltung eines \\ sozial-ökologischen Wandels
}

\author{
Kathrin Niedermoser
}

(C) Der/die Autor(en) 2017. Dieser Artikel ist eine Open-Access-Publikation.

Zusammenfassung Trotz der evidenten Auswirkungen des Klimawandels bzw. der aktuellen Klimapolitik auf das Feld der Arbeitswelt spielen Gewerkschaften als zentrale Akteure in diesem Bereich in den aktuellen sozial-ökologischen Debatten meist eine untergeordnete Rolle. Obwohl sich in Österreich kaum eine eigenständige gewerkschaftliche Klimapolitik erkennen lässt, zeigt sich, dass Gewerkschaften entlang der unterschiedlichen Branchen Umwelt- und Klimathemen aufnehmen. Der Beitrag gibt einen knappen historischen Abriss über das konfliktive Verhältnis der organisierten österreichischen ArbeiterInnenbewegung zu Umweltfragen. Zudem werden aktuelle Debatten und Entwicklungen bezüglich der Positionierung von Gewerkschaften zu Umwelt- und Klimathemen skizziert. Im Vordergrund steht die Frage, welche konkreten Konfliktlinien der Zugang von Gewerkschaften zu ökologischen Fragen in sich birgt und welche konkreten Ansatzpunkte es in den österreichischen Gewerkschaften aktuell gibt, um ökologischen Problemen zu begegnen.

Schlüsselwörter Gewerkschaften · Klimawandel · Nachhaltigkeit · Arbeit · Umwelt

\section{"If we do not grow any longer, how do we redistribute?"}

The role of trade unions within social-ecological transformation

\footnotetext{
Abstract Despite the evident impact of climate change as well as the current climate policy on the world of labour, trade unions - being key actors in this field - in most cases only play a marginal role in current social-ecological debates. Although an independent trade union climate policy in Austria hardly exists, trade unions take

K. Niedermoser $(\square)$

Institut für Politikwissenschaft, Universität Wien, Universitätsstraße 7/2. Stock, 1010 Wien, Österreich

E-Mail: kathrin.niedermoser@univie.ac.at
} 
up environmental and climate issues along the different branches of industry. This paper provides a concise historic outline of the conflictual relationship of the organised Austrian labour movement towards environmental questions. Furthermore, current debates and developments regarding the positioning of trade unions to environmental and climate issues are outlined. Special emphasis is given to the question, what specific lines of conflict are involved in the approach of trade unions towards ecological questions and what specific starting points currently exist in the Austrian trade unions to counter ecological problems.

Keywords Trade unions $\cdot$ Climate change $\cdot$ Sustainability $\cdot$ Labour $\cdot$ Environment

\section{Einleitung}

Der Klimawandel gehört zweifelsohne ,zu den international am heftigsten umstrittenen Konfliktfeldern“ (Scheffran 2004, S. 184). Trotz der evidenten Auswirkungen des Klimawandels und der aktuellen Klimapolitik auf das Feld der Arbeitswelt (vgl. Rosemberg 2010; Lebensministerium 2012) spielen Gewerkschaften als zentrale Akteure in diesem Bereich in der aktuellen Klimapolitik meist eine untergeordnete Rolle. Wurden Gewerkschaften auf der „Rio-Konferenz“ 1992 noch als zentrale Akteure beim Umbau zu einer nachhaltigen Gesellschaft betrachtet (vgl. UNEP 1992), bleibt deren Rolle im aktuellen politischen und wissenschaftlichen Diskurs meist unbeachtet. Dieser Umstand lässt sich einerseits durch eine generelle Schwächung von Gewerkschaften vor dem Hintergrund der neoliberalen Wende und den Auswirkungen von Globalisierungsprozessen erklären (vgl. Dörre 2007), ist jedoch auch Ausdruck davon, dass die (Sozial-)Wissenschaft(en) Gewerkschaften zunehmend aus dem Blickfeld verloren haben. Hinzu kommt, dass Gewerkschaften zu Umweltfragen traditionell ein ambivalentes Verhältnis haben und mehr als Kontrahent denn als BündnispartnerInnen wahrgenommen werden. In Österreich lässt sich kaum eine eigenständige gewerkschaftliche Klimapolitik erkennen, sondern vielmehr ein klimapolitischer Korporatismus feststellen (vgl. Brand und Pawloff 2014). Dennoch zeigt sich, dass Gewerkschaften in den unterschiedlichen Branchen Umwelt- und Klimathemen aufnehmen.

Der folgende Beitrag gibt einen knappen historischen Abriss über das konfliktive Verhältnis der organisierten österreichischen ArbeiterInnenbewegung zu Umweltfragen. Zudem werden aktuelle Debatten und Entwicklungen bezüglich der Positionierung von Gewerkschaften zu Umwelt- und Klimathemen skizziert. Im Vordergrund steht die Frage, welche konkreten Konfliktlinien der Zugang von Gewerkschaften $\mathrm{zu}$ ökologischen Fragen in sich birgt und welche konkreten Ansatzpunkte es in den österreichischen Gewerkschaften aktuell gibt, um ökologischen Problemen zu begegnen. Der Beitrag entstand im Rahmen des Forschungsprojektes „TRA- 
FO LABOUR“. ${ }^{1}$ Das transdisziplinäre Projekt wurde in enger Zusammenarbeit mit der Produktionsgewerkschaft (PRO-GE), der Gewerkschaft Bau-Holz (GBH), der Gewerkschaft vida sowie der Arbeiterkammer Wien durchgeführt. Die zentrale Fragestellung war, welche Hindernisse, aber auch Anknüpfungspunkte sich für Gewerkschaften bei der Gestaltung einer sozial-ökologischen Transformation ergeben. Neben einer Diskursanalyse von wissenschaftlichen Beiträgen und Dokumenten aus den Gewerkschaften wurden qualitative ExpertInnen-Interviews und StakeholderDialoge durchgeführt. Für den vorliegenden Beitrag wurden zudem ExpertInnenInterviews aus dem Jahr 2011 berücksichtigt.

\section{Die historische ArbeiterInnenbewegung und Umweltfragen}

Ausgehend von der Annahme, dass es sich bei Gewerkschaften um historisch gewachsene Organisationen handelt, deren Umgang mit ökologischen Fragestellungen eine lange und widersprüchliche Geschichte hat, erscheint es ergiebig ,das Entstehen verfestigter Denkweisen und tradierter Praktiken nachzuzeichnen und dadurch deren - mögliche - Wirksamkeit in der Gegenwart transparent zu machen“ (Sandner 1999, S. 12). Aus historischer Sicht gestaltet sich der Umgang der ArbeiterInnenbewegung mit ökologischen Fragen schwierig und ist von Kontinuitäten und Brüchen geprägt. Der diesbezügliche Forschungsstand für Österreich muss als spärlich bezeichnet werden. Während für Deutschland und England einige dokumentierte Fälle vorliegen, die die Konfliktlinie zwischen ArbeiterInnenbewegung und ökologischen Problemstellungen nachzeichnen (vgl. Lackner 1990; Schramm 1990; Andersen 1990), gibt es hierzu betreffend Österreich kaum Forschungsergebnisse.

Die oftmals attestierte theoretische Schwäche der historischen ArbeiterInnenbewegung bezüglich „Umweltfragen“ hält einer näheren Betrachtung jedenfalls nicht stand. Vielmehr war diese bereits zum Zeitpunkt ihrer Entstehung mit konkreten ökologischen Fragen konfrontiert. Schon in den klassischen theoretischen Schriften des 19. Jahrhunderts finden sich grundsätzliche Überlegungen zum Verhältnis von Mensch und Natur (vgl. u. a. Gärtner 1990; MEW 23; Schramm 1990). Ebenso lässt sich bei den TheoretikerInnen der österreichischen ArbeiterInnenbewegung ein grundsätzliches Bewusstsein über die negativen Auswirkungen von Luft- und Wasserverschmutzungen feststellen. Victor Adler etwa zog die Schlussfolgerung, dass der ,Zustand der Faktoren von welchen die Volksgesundheit in erster Linie beeinflusst wird: Luft und Wohnung, Trinkwasser und Boden, Lebenshaltung und Nahrung“ (Adler 1924, S. 69) vermehrt untersucht werden sollte. Hygiene, Gesundheit und Wohnverhältnisse wurden mit ökologischen Fragen verknüpft und als Teil der „sozialen Frage“ betrachtet. Sandner beschreibt diese „Krise der menschlichen Natur“ (Sandner 1999, S. 9) als den ,Angelpunkt sozial- und kulturpolitischer Reformbestrebungen“" (ebd.) innerhalb der sozialdemokratischen ArbeiterInnenbewegung zu

\footnotetext{
1 Das Projekt ,TRAFO LABOUR - The Role of Trade Unions and Workers' Interests in the Social-Ecological Transformation towards a Climate-Friendly Society. The Case of Austria“, wurde aus Mitteln des österreichischen Klima- und Energiefonds gefördert und im Rahmen des Programmes „ACRP $6^{\text {th }}$ Call“" von 2014-2016 durchgeführt.
} 
Beginn des 20. Jahrhunderts. Der historischen ArbeiterInnenbewegung mangelte es also durchaus nicht an einem grundsätzlichen (theoretischen) Verständnis über den Stoffwechsel zwischen Mensch und Natur im gesellschaftlichen (Re-)Produktionsprozess und den sich daraus ergebenden Auswirkungen für die Umwelt. Vielmehr ist es der Widerspruch zwischen theoretischen Standpunkten und der alltäglichen Praxis innerhalb der ArbeiterInnenbewegung, der anhand des Umgangs mit ökologischen Fragen deutlich wird. „Es gilt vielmehr zu fragen, auf welchen konkreten Erfahrungsgebieten auch ArbeiterInnen sowie ihre VordenkerInnen und Organisationen die negativen Umweltfolgen der Industrialisierung wahrzunehmen und darauf politisch zu reagieren vermochten“ (Linse 1993, S. 120).

In historischer Betrachtung scheinen vier Diskurse von besonderer Relevanz. Dies ist erstens der widersprüchliche Zugang der ArbeiterInnenbewegung zu technologischem Fortschritt, dessen Bandbreite von „Technikfetisch“ bis zur „Maschinenstürmerei“ reichte. Zweitens zeigt sich, dass sich im Zusammenhang mit ökologischen Problemstellungen bereits im 19. Jahrhundert die Frage von Standort und Wettbewerb stellte (vgl. Schramm 1990). Das daran anknüpfende „Arbeitsplatzargument“ stellt jenen roten Faden dar, der in Konfliktfällen immer wieder auftaucht. Drittens zeigt sich auch, dass das Umweltbewusstsein der ArbeiterInnenbewegung unter anderem in der Wahrnehmung von Natur als Ort der Erholung (vgl. Lackner 1990) zum Ausdruck kam, was durchaus als „Bestandteil der Emanzipation der Arbeiterklasse und der sozialistischen Bewegung“ (Harmsen 1990, S. 90) betrachtet werden kann. Vor allem der ,Zug in's Freie“ (ebd.), welcher Natur zum Ort macht, wo Freizeit und Erholung gestaltet werden, findet sich bereits in der frühen ArbeiterInnenbewegung und schlug sich später auch in der Gründung von Vereinen und Organisationen wie beispielsweise der „Naturfreunde“ nieder, die die Natur nicht nur erschließen, sondern auch schützen wollten (vgl. Linse 1993). Viertens und am schwerwiegendsten war für die historische ArbeiterInnenbewegung in Bezug auf ökologische Fragen jedoch zweifelsohne die Kluft bezüglich der Wahrnehmung von inner- und außerbetrieblichen Umweltbelastungen. Der „Arbeitsschutz“ wurde im Laufe der Weiterentwicklung der industriellen Produktion, neben der Wohnungsfrage und allgemeinen medizinisch/hygienischen Aspekten, zu einem zentralen Bestandteil gesundheitspolitischer Fragen (vgl. Linse 1993). Dies erklärt sich unter anderem damit, ,dass die unmittelbaren Erfahrungen mit den Umweltbelastungen am Arbeitsplatz die außerbetrieblichen Umweltbelastungen oft als vernachlässigbar erscheinen" (Glatz 1984, S. 129) ließen. Das Themenfeld der inner- und außerbetrieblichen Umweltbelastungen hat sich seit dem Beginn der Industrialisierung zweifelsohne stark verändert und vor dem Hintergrund globalisierter Konsum- und Produktionsketten eine neue Dimension erhalten. Während innerbetriebliche Umweltbelastungen in Westeuropa seitens der Gewerkschaften erfolgreich eingeschränkt werden konnten, gehört die gesundheitliche Gefährdung von Beschäftigten in vielen anderen Teilen der Welt nach wie vor zum Alltag. Das Konfliktfeld inner- und außerbetrieblicher Umweltbelastungen ist also nicht verschwunden, sondern wurde im Rahmen der internationalisierten Arbeitsteilung globalisiert, wobei aus westeuropäischer Sicht sowohl die inner- als auch die außerbetrieblichen Umweltbelastungen zu großen Teilen externalisiert wurden. 
Aufbauend auf diesen Diskursen und konkreten Erfahrungen muss den Organisationen der ArbeiterInnenbewegung seit jeher ein ambivalentes Verhältnis zu ökologischen Fragen attestiert werden. Für die jüngere Vergangenheit sind es vor allem die Konflikte rund um die Kraftwerke Zwentendorf und Hainburg, die das Verhältnis der österreichischen Gewerkschaften bezüglich ökologischer Fragen mit nachhaltiger Wirkung bis in die Gegenwart prägen und daher im Folgenden einer näheren Betrachtung unterzogen werden.

\section{Das Trauma von Zwentendorf und Hainburg}

Wie in den meisten anderen westeuropäischen Ländern wurden ökologische Fragen auch in Österreich zu Beginn der 1970er-Jahre Gegenstand gesellschaftlicher Auseinandersetzungen. Dieser Umstand fand auch innerhalb der organisierten ArbeiterInnenbewegung seinen Ausdruck. So wurde bereits 1973 in der Arbeiterkammer ein „Umweltpolitisches Referat“ gegründet, und der Umweltbegriff fand 1975 erstmals Einzug in das Programm des Österreichischen Gewerkschaftsbundes (ÖGB) und erlangte in den darauf folgenden Jahren zunehmend an Bedeutung (vgl. Glatz 1984). Die Konflikte rund um die geplante Inbetriebnahme des Atomkraftwerkes in Zwentendorf (1978) und das Bauvorhaben eines Wasserkraftwerkes in Hainburg (1984) markierten nicht nur den Höhepunkt umweltpolitischer Auseinandersetzungen in Österreich, sondern müssen auch ,als Symptome gesellschaftlichen Wandels“ (Natter 1987, S. 151) betrachtet werden.

Der vor dem Hintergrund der Ölkrise 1973/74 entstandene Energieplan von 1976 sah vor, „Erdöl durch Kohle, Kernenergie, Wasserkraft und Erdgas zu ersetzen“ (Winkler-Rieder 1997, S. 623). ,Die AKWs sollten billigen Strom produzieren, wobei es anfänglich vor allem die Industrie war, die ein billiges Substitut für Erdöl suchte. Die Gewerkschaften schlossen sich diesem Kurs aus wachstums- und beschäftigungspolitischen Gründen an.“ (ebd.) In beiden Fällen positionierte sich der ÖGB nicht nur - über die Sozialpartnerschaft in einem ,klassenübergreifenden Charakter“ - (Natter 1987, S. 166) eindeutig auf Seiten der Kraftwerksbauer, sondern nahm auch eine „,besonders aktive Rolle“ (ebd.) ein.

Bundeskanzler Kreisky bescheinigte nach der knapp verlorenen Volksabstimmung gegen das AKW Zwentendorf, dass die „Gewerkschaftsfraktion und der persönliche Einsatz von ÖGB-Präsident Benya [...] wesentlich zu diesem knappen Ergebnis beigetragen“ (APA-Meldung, 6. November 1978) habe.

Auch der Bau des Wasserkraftwerkes in der Hainburger Au wurde vom Österreichischen Gewerkschaftsbund und der Bundesarbeiterkammer befürwortetet. Damit geriet die institutionalisierte ArbeiterInnenbewegung nach Zwentendorf zum zweiten Mal innerhalb weniger Jahre in Konflikt mit der in den Kinderschuhen steckenden Umweltbewegung. Sowohl Gewerkschaften als auch BetriebsrätInnen brachten sich aktiv in den Konflikt rund um das Wasserkraftwerk ein. Nachdem die Rodungsarbeiten im Dezember 1984 vorerst gestoppt wurden, kündigte der ehemalige Zentralsekretär der Gewerkschaft Metall - Bergbau - Energie und Klubchef der SPÖ, Sepp Wille, im Parlament Arbeiterdemonstrationen gegen den Baustopp an. Zudem wurde eine BetriebsrätInnenkonferenz unter dem Motto „Pro Hainburg“ abgehalten (vgl. 
Baschnegger 1985). Für Empörung auf Seiten der KraftwerksgegnerInnen sorgte die Aussage von ÖGB-Vizepräsident Johann Gassner, welcher den Wunsch aussprach, „die Exekutive möge nun sorgen, daß die Arbeiter in der Au bald ihre Tätigkeit aufnehmen können" (APA-Meldung, 17. Dezember 1984), sowie die Aussage des Betriebsrates des Bauunternehmens Universale, Josef Kerschbaum, der ankündigte, „daß die Belegschaft übermorgen dafür sorgen würde, daß die Au-Besetzer das Neue Jahr bereits bei sich zu Hause verbringen“" (ebd.).

Die Auseinandersetzung in und rund um die Au wurde durch den so genannten „Weihnachtsfrieden“ vorerst beendet. Dieser wurde durch den damaligen Bundeskanzler Sinowatz ausgerufen. In weiterer Folge bestätigte der Verwaltungsgerichtshof, welcher am 3. Jänner einem Beschwerdeantrag von WWF und Grundstückseigentümern stattgab und somit weitere Rodungsarbeiten untersagte (vgl. Nenning/ Huber 1985, S. 163), den Stopp des Bauvorhabens. Das von 4.-11. März 1985 durchgeführte „Konrad-Lorenz-Volksbegehren“ gegen das Kraftwerk in Hainburg wurde von 353.900 Menschen unterschrieben (vgl. Hainburger-Au 2011). Der endgültige Schlussstrich wurde im Oktober 1985 gezogen, als eine von der Bundesregierung eingesetzte „Ökologiekommission“ ein Donaukraftwerk am Standort Hainburg ablehnte (vgl. APA-Meldung, 31. Oktober 1985).

Doch auch innerhalb der Gewerkschaften regte sich Widerstand gegen die offizielle Linie bezüglich beider Kraftwerke. Im Kontext von Zwentendorf entstand 1977 die fraktionsübergreifende Initiative „GewerkschafterInnen gegen Atomenergie“ (vgl. Leisch 2009, S. 104). Diese forderte „,breite Diskussion und freie Meinungsbildung in Betrieben, Fach- und Ortsgruppen ...“ (ebd.) Im Zusammenhang mit Hainburg erfolgte die Gründung der „Plattform Gewerkschafter gegen Hainburg“ (vgl. Dworczak 1985, S. 106), welche sich auf die ,lebensbejahende Tradition“ (ebd.) der ArbeiterInnenbewegung berief. Im Prominentenkomitee unter der Führung von Konrad Lorenz befand sich zudem Günter Nenning, damaliger Vorsitzende der Gewerkschaftssektion Journalisten im ÖGB, der eine zentrale Rolle unter den KraftwerksgegnerInnen einnahm. Zweifelsohne handelte es sich in beiden Fällen um oppositionelle Stimmen. Diese „Geschichte von Unterlegenen“ (Hermand 1991, S. 17, zit. nach: Sandner 1999, S. 49) zieht sich durch das gesamte Politikfeld, da VertreterInnen von ökologischen Ansätzen meist im ,Widerspruch zum mainstream des jeweiligen politischen Lagers“ (Bramwell 1989, S. 13, zit. nach: Sandner 1999, S. 49) standen. Aktuell zeigt sich dies beispielsweise an der Fraktion der Alternativen und Grünen GewerkschafterInnen (AUGE/UG), die als kleine oppositionelle Gewerkschaftsströmung die weitreichendsten Überlegungen und Kritikpunkte bezüglich der gewerkschaftlichen Praxis zu ökologischen Fragestellungen einnimmt (vgl. u. a. AUGE/UG 2009).

Zusammenfassend lassen sich drei zentrale Dimensionen bezüglich der Rolle der Gewerkschaften in Bezug auf die Konflikte rund um Zwentendorf und Hainburg feststellen. Erstens die Herangehensweise, ein gemeinsames Interesse mit der Industrie zu verfolgen; daran anknüpfend die Frage von Demokratie und Partizipation und drittens der traditionelle Konflikt „Arbeitsplätze vs. Umweltschutz“. Diese drei Konfliktfelder werden im Folgenden näher dargestellt.

Die Teilgewerkschaften bzw. der ÖGB agierten sowohl im Fall von Zwentendorf als auch in Hainburg in enger Kooperation mit der Wirtschaftskammer und der 
Industriellenvereinigung. So titelte eine Presseaussendung der Sozialpartner bereits zu Beginn des Jahres 1984 mit „Arbeitnehmer und Arbeitgeber einig. Hainburg wird gebraucht“" (APA-Meldung, 19. Jänner 1984) und sah in einer Verzögerung des Baubeginns ,nachteilige Folgen für Arbeitnehmer, die beteiligten Unternehmen, aber auch die Konsumenten“ (ebd). Die KraftwerksgegnerInnen stellten dem die Gefährdung der „Gesundheit und Sicherheit der Bevölkerung“ durch die Kernenergie (APA-Meldung, 17. Mai 1976) gegenüber. Auch im Falle von Zwentendorf ging der ÖGB ein Bündnis mit der Industriellenvereinigung, Siemens und der Wirtschaftskammer ein, um ein „Ja“ für Zwentendorf zu propagieren (vgl. Leisch 2009, S. 106). Seitens der GegnerInnen wurde in beiden Fällen kritisiert, dass sich die Gewerkschaften den Interessen der österreichischen Energielobby unterordnen würden. So forderte die ,Plattform Gewerkschafter gegen Hainburg“ eine inhaltliche Orientierung ,gegen die Profitinteressen der E-Wirtschaft“ (Dworczak 1985, S. 106) seitens des ÖGB ein. Das gemeinsame Interesse, welches die Gewerkschaften dabei in den Mittelpunkt stellten, muss vor dem Hintergrund der Sozialpartnerschaft und ihrem „konstitutiven Element“ (Glatz 1984, S. 126) - der Wachstumslogik - betrachtet werden. Gerade diese „Wachstumsillusion“ (Blau und Häupl 1980, S. 167) wurde jedoch zunehmend, auch über die Ökologiebewegung hinaus, in Frage gestellt. Ebenso der Kompromiss, der nach 1945 in Form der Sozialpartnerschaft geschmiedet wurde. So erlebte dieser sowohl mit Zwentendorf als auch mit Hainburg eine „nachhaltige Erschütterung“ (Lackner 1990, S. 213), wobei diesbezüglich auch „das weit verbreitete, aus unterschiedlichen Quellen gespeiste Unbehagen mit den traditionellen politischen Parteien und Wirtschaftsverbänden in einem populistischen ,Gegen-die-da-oben““ (Natter 1987, S. 163) deutlich wurde. Paul Blau sieht in der Auflehnung gegen Hainburg und Zwentendorf den Beweis für eine ,fortschreitende Emanzipation von Obrigkeitshörigkeit“ (Blau 1999, S. 162), wobei die Gewerkschaften auch als Teil des „Establishments“ (ebd., S. 163) wahrgenommen wurden. Dieselbe Analyse zogen AktivistInnen der „Nein zu Zwentendorfbewegung“ (vgl. Leisch 2009).

Die Auseinandersetzungen um die beiden Kraftwerke Hainburg und Zwentendorf belebten auch den ,alten“ Konflikt „Arbeitsplätze vs. Umweltschutz“ neu. Bereits 1983 warnte die „Vereinigung der industriellen Bauunternehmer Österreichs“ davor, „dass eine Verzögerung des Kraftwerkbaues die Arbeitslosigkeit unter den Bauarbeitern weiterhin verschärfen würde“ (APA-Meldung, 10. August 1983). Der Zentralbetriebsrat der VÖEST-Alpine AG, Franz Schwarz, argumentierte, dass der Bau des Kraftwerkes Hainburg in der VÖEST rund 800 Arbeitsplätze für ein Jahr absichere und ein „Nein“ zu Hainburg 300 Arbeitsplätze unmittelbar gefährden würde (vgl. APA-Meldung, 13. Dezember 1984). Auf einem Transparent auf einer ProHainburg-Demo am Heldenplatz am 17. Mai 1984 war der Spruch zu lesen: „Die Arbeitsplätze müssen Vorrang haben“ (Hesoun und Pötschacher 1985, S. 74). Auch innerhalb der Diskussion rund um das AKW Zwentendorf wurde seitens der BefürworterInnen mit den neu entstehenden Arbeitsplätzen argumentiert bzw. im Falle eines „Neins“ bei der Volksabstimmung vor „Arbeitsplatzverlust“ gewarnt.

Die skizzierten Erfahrungen in den 1970er und 1980er Jahren sind in den österreichischen Gewerkschaften nach wie vor präsent und werden als „Traumata“ (Interview 02) bezeichnet. Dennoch lässt sich rund um den Jahrtausendwechsel 
eine Annäherung zwischen Gewerkschaften und der Umweltbewegung feststellen. Die über die Auswirkungen der Globalisierung entstandene Verbindungslinie zwischen Umweltthemen und Gewerkschaften (vgl. ILO 2002; Gregory et al. 1999) manifestierte sich auch in Österreich an Kampagnen wie „STOP-GATS“ oder der „Sozialforumsbewegung“.

Zudem lässt sich für diesen Zeitraum auch eine verstärkte inhaltliche Annäherung feststellen, wie Beiträge aus den Gewerkschaften aufzeigen, die sich mit Fragen der Globalisierung (vgl. Blau 1998) und strukturellen Veränderungen der Arbeitswelt und deren Auswirkungen auf ökologische Fragen (vgl. Blau et al. 1997; Ritt 1998; Littig und Grießler 2001) befassten.

Vor dem Hintergrund der aktuellen Klimadebatte und den anhaltenden Folgen der Finanz- und Wirtschaftskrise 2008/2009 erlangten diese Allianzen erneut an Bedeutung. Ein Beispiel hierfür stellt die Kampagne „Umwelt + Bauen“ dar (vgl. Umwelt + Bauen 2011): eine Kooperation zwischen der Gewerkschaft Bau-Holz (GBH), dem ÖGB, der Umwelt-NGO GLOBAL 2000, der Bundesarbeiterkammer sowie der Wirtschaftskammer Österreich und VertreterInnen aus dem Bereich der Bauindustrie, welche 2010 ins Leben gerufen wurde. Erklärtes Ziel ist es, die Öffentlichkeit von der Notwendigkeit nachhaltiger Investitionen zu überzeugen, die thermische Sanierung in Österreich voranzutreiben und damit Arbeitsplätze in der Bauindustrie zu schaffen (vgl. ebd., S. 3). Bemerkenswert an diesem Bündnis ist, dass mit der GBH und GLOBAL 2000 einstige Konfliktparteien an einem Strang ziehen. Vor allem die GBH hat hierzu in den letzten Jahren einige Initiativen entwickelt und greift das eigene Image als „Betonierer“ (ebd., S. 1) kritisch auf. Ein weiteres Beispiel für diese Annäherung ist das zivilgesellschaftliche Bündnis ,Wege aus der Krise“. Auch in diesem Kontext arbeiten Gewerkschaften und Umwelt-NGOs eng zusammen, um sozial-ökologische Alternativen zur vorherrschenden Krisenpolitik aufzuzeigen (vgl. Wege aus der Krise 2014).

\section{Zwischen Krise, Wettbewerb und einem guten Leben für alle?}

Ein Blick in die aktuellen Positionen und Beschlüsse des ÖGB und einzelner Gewerkschaften zeigt, dass Umweltprobleme im Allgemeinen bzw. der Klimawandel im Besonderen in unterschiedlicher Ausprägung als Problem identifiziert und aus unterschiedlichen Perspektiven diskutiert werden. So spricht der ÖGB in seinem Grundsatzprogramm von der Notwendigkeit einer ,Ökologisierung aller Lebensbereiche“ (ÖGB 2013, S. 105), die Gewerkschaft der Privatangestellten, Druck, Journalismus, Papier (GPA-djp) nennt als Ziel ,eine sozial und ökologisch verträgliche wirtschaftliche Entwicklung“ (GPA-djp 2015, S. 151), die Produktionsgewerkschaft (PRO-GE) nennt neben sozialer Gerechtigkeit und Frieden eine ,gesunde Umwelt“ (PRO-GE 2013, S. 6) als Grundpfeiler für eine Gesellschaft, in der ArbeitnehmerInnen einen besonderen Stellenwert haben, die Gewerkschaft Bau-Holz nennt Nachhaltigkeit dezidiert als gesellschaftspolitisches Ziel (vgl. GBH 2014, S. 3). und die Gewerkschaft vida sieht im Klimawandel eine der „größten globalen Bedrohungen für Mensch und Umwelt" (vida 2010, S. 23). Die Gewerkschaften greifen dabei meist Problemfelder auf, die sich aus der aktuellen ökologischen Krise für 
die gewerkschaftliche Praxis ergeben. Von zentraler Bedeutung sind die Bereiche Verkehr und Energieversorgung, Maßnahmen zur thermischen Sanierung, aber auch weiterreichende politische Forderungen zur internationalen Handelspolitik oder den Auswirkungen der Globalisierung. Zudem wurde auf dem ÖGB-Kongress 2013 ein umfassendes Konzept für eine „ökologische Steuerreform“ (ÖGB 2013, S. 106) beschlossen.

Grundsätzlich lässt sich festhalten, dass ökologische Themen von den österreichischen Gewerkschaften durchaus aufgenommen bzw. thematisiert werden. Auch wenn sich dies oftmals auf die programmatische Ebene in Form von Beschlüssen oder Dokumenten beschränkt, lässt sich feststellen, dass über Themen wie etwa der Ausbau von Bahnstrecken und des öffentlichen Verkehrs, die Ausweitung der LKWMaut oder das Bekenntnis zu einer Energiewende (vgl. ebd.) zentrale ökologische Herausforderungen thematisiert werden, obwohl diese für einzelne Branchen weit reichende Konsequenzen mit sich bringen. Inwiefern diese Beschlüsse Einzug in die tatsächliche Politikgestaltung von Gewerkschaften haben werden, bleibt abzuwarten. Als zentrales Hindernis dabei müssen die branchenspezifischen Auswirkungen von klimafreundlichen Maßnahmen gesehen werden. Dies betrifft neben der Automobil(zulieferer)industrie vor allem die Schwerindustrie wie beispielsweise die voestalpine AG.

\section{Wachstumslogik, Wettbewerb und Standort}

Obwohl sich eine zunehmende Sensibilisierung innerhalb der Gewerkschaften in Bezug auf ökologische Fragestellungen feststellen lässt, bleibt mit dem ,widersprüchlichen Verhältnis zwischen Wachstum, Arbeitsplatzerhalt und Umverteilung auf der einen und dem Klimaschutz auf der anderen Seite“ (Walk und Brunnengräber 2000, S. 70) die zentrale Konfliktlinie weiterhin bestehen. Dies stellt insofern einen wesentlichen Angelpunkt dar, als dass die Ursachen des Klimawandels eng mit einem Entwicklungsmodell verbunden sind, das auf Wachstum und Beschleunigung ausgerichtet ist und dessen materielle Basis auf fossilen Energieträgern beruht (vgl. Brunnengräber et al. 2008; Passadakis und Müller 2009). Die Politik der Gewerkschaften ist nach wie vor von der Logik dieses Wachstumsmodells durchdrungen und bestimmt ganz wesentliche Felder der gewerkschaftlichen Praxis, wie die Schaffung von Arbeitsplätzen oder sozial- und verteilungspolitische Positionierungen. So wird „eine wachsende Wirtschaft“ (Delapina 2011, S. 6) als Vorrausetzung für ,wachsende Einkommen für alle“ (ebd.) betrachtet, während in Zeiten der Stagnation „Einkommenssteigerungen für eine Gruppe“ (ebd.) nur möglich sind, ,,wenn anderen etwas weggenommen wird“ (ebd.).

Besonders deutlich wird hierbei die Verzahnung eines wachstumsorientierten Entwicklungsmodells mit einem „korporativen keynesianischen Klassenkompromiss“ (Altvater 2009, S. 98), der in Österreich in Form der Sozialpartnerschaft eine wesentliche Politikstrategie der Gewerkschaften darstellt. So wird diese vom ÖGB als ,auf gesellschaftlichen Interessensausgleich ausgerichtete Kultur“ (ÖGB 2011, o. S.) bezeichnet, die ,einen nicht unwesentlichen Teil dazu beigetragen hat, dass Österreich in der Zweiten Republik auf wirtschaftlichem und sozialem Gebiet eine 
hervorragende Entwicklung zu verzeichnen hatte“ (ebd.). Hinter dieser „Produktivitätssteigerungs-Politik“ (Lackner 1990, S. 213) verbirgt sich „,eine Ausweitung der Bezugspunkte und Perspektiven gewerkschaftlicher Interessenspolitik“ (Tálos 1997, S. 438), die seit den 1950er-Jahren ,zu einem Kernpunkt des Selbstverständnisses der österreichischen Gewerkschaften geworden“ (ebd.) ist. „Die Gewerkschaften gehen dabei von der Erkenntnis aus, dass die Steigerung und Verbesserung der Produktivität eine der Voraussetzungen für weitere Erhöhungen des Lebensstandards des Volkes sind“ (Tálos 1997, S. 438). Die an das Wachstumsmodell angelehnte Steigerung der „Massenkaufkraft“ als wesentliche ökonomische Größe und die propagierten „Aufsteigermodelle“ als gesellschaftliches Konzept geraten neben ökologischen auch aus sozialen Gründen ins Wanken. Die gesellschaftliche Funktion von Konsum, die sich nach dem 2. Weltkrieg als „Lebensstil“" in den Industrieländern etabliert hat (vgl. Altvater 2009, S. 100) und sich zur ,imperialen Lebensweise“ (Brand und Wissen 2011, S. 80) entwickelt hat, stellt Gewerkschaften vor neue Herausforderungen, da diese meist als Ausdruck erfolgreicher sozialpartnerschaftlicher Verteilungspolitik gedeutet wird. Zugleich stehen Gewerkschaften vor dem Dilemma, dass das nach 1945 anvisierte und teilweise auch erreichte Wohlstandsmodell für breite Teile der Gesellschaft dazu geführt hat, dass ,,viele Menschen an den gegenwärtigen un-nachhaltigen Verhältnissen teilhaben wollen“ (Brand 2008, S. 142). Eine Absage an diese Verhältnisse würde nicht nur Verzicht bedeuten, sondern neben dem vorherrschenden Wachstumsmodell auch das von Gewerkschaften propagierte Wohlstandsmodell in Frage stellen.

Die sozialpartnerschaftlich organisierte und auf Wachstum orientierte Politik der letzten Jahrzehnte ist massiv unter Druck geraten. Der Rückgang der Lohnquoten und das Auseinanderdriften zwischen Produktivität und Lohnentwicklung (vgl. u. a. AK Oberösterreich 2010) zeigen auf, dass der verteilungspolitische Effekt über stetige Wachstumsraten zurückgeht. Altvater sieht als Grund dafür die Tatsache, dass Faktoren, die Wachstum steigern, auch Ungleichheiten fördern, da Wachstum auf Kapitalakkumulation basiert und somit auch die Aneignung von Überschüssen beinhaltet (vgl. Altvater 2009, S. 92). Aufgrund der ökologischen Komponente von Wachstum, das auf die Ausbeutung von Rohstoffen und die Nutzung natürlicher Senken angewiesen ist, geraten Gewerkschaften aufgrund ihrer wachstumsorientierten Politik in Konflikt zu Umwelt- bzw. Klimaschutz. So bezeichnete ein Interviewpartner die österreichische Klimastrategie als „Wachstumsbremse“ (Interview 01) und wies in diesem Zusammenhang auf die Korrelation zwischen Wirtschaftswachstum und Arbeitslosigkeit hin (ebd.). Auch jene GewerkschafterInnen, die eine grundsätzlich kritische Einstellung zum Wachstumsmodell offenlegten, verwiesen auf diesen Zusammenhang (vgl. Interview 04/05). Als möglicher Ausweg wird die Entkoppelung von Wirtschaftswachstum und Ressourcenverbrauch mittels Effizienzsteigerungen skizziert (vgl. GPA-djp 2015, S. 137) bzw. auch ein ,qualitatives“ (Interview 02/04/05) oder ein ,nachhaltiges Wachstum“ (ÖGB 2013, S. 81; GPA-djp 2015, S. 126) angestrebt. Außerdem sehen die Gewerkschaften über eine neue Verteilung der Produktivität durch die Verkürzung der Arbeitszeit (vgl. Interview 05) bzw. über die verstärkte Besteuerung von Vermögen (vgl. ÖGB 2013) eine Möglichkeit, dem Zusammenhang zwischen Wachstum und Umweltzerstörung entgegenzuwirken, indem ein neues „Wachstums- und Verteilungsmodell“ (PRO-GE 
2013, S. 8) entwickelt werden soll. Als wesentliche Elemente hierbei werden neben „öffentlichen Investitionen in eine nachhaltige, soziale und ökologische Infrastruktur“, einer Energiewende und „einer koordinierten europäischen Industriepolitik“ eine „kooperative Wirtschaftspolitik“, um die innereuropäischen Ungleichgewichte abzubauen, und die Regulierung des Finanzsektors inklusive einer Finanztransaktionssteuer genannt (vgl. ebd.).

Eng verknüpft mit der Frage des Wachstums sind Wettbewerbs- und Standortlogik. Die InterviewpartnerInnen berichteten im Zusammenhang mit $\mathrm{CO}_{2}$-Gratiszertifikaten von Abwanderungsdrohungen einzelner Unternehmen (vgl. Interview 02). Die negativen Auswirkungen der Klimapolitik finden sich auch in den Stellungnahmen der Sozialpartner wieder. Darin bekennen sich die Sozialpartner zwar zu „einer Verringerung der Treibhausgase“ (Österreichische Sozialpartner 2008, S. 1), räumen jedoch ein, dass daraus resultierende ,negative Effekte auf den Wirtschaftsstandort" (ebd.) möglichst verhindert werden sollten. Diese strategische Allianz mit der Arbeitgeberseite lässt sich auch durch einen Mangel an Alternativen erklären, da die Handlungsspielräume der Gewerkschaften aufgrund der skizzierten sozialpartnerschaftlichen Grundausrichtung sehr beschränkt sind. Während die Industrie stets eine ,exit-Option“ (Standortverlagerungen) im Ärmel hat, haben Gewerkschaften in diesem „Politikfeld lediglich die loyality- und voice-Option, die sich beide im Bündnis mit der Industrie stärken lassen“ (Walk und Brunnengräber 2000, S. 70). Konkret bedeutet dies, dass Gewerkschaften im Sinne der Standort- und Wettbewerbslogik Unternehmensstrategien mittragen oder versuchen, über sozialpartnerschaftliche Strukturen Verbesserungen zu verhandeln. Diese bewegen sich meist innerhalb der Logik vorherrschender Modernisierungsstrategien, die vor allem auf technologische und marktbasierte Lösungen setzen (vgl. Felli 2014) und selten grundsätzlichere strukturelle Veränderungen forcieren.

\section{Arbeitsplätze und Umweltschutz}

Wie eingangs erwähnt, prägte die Konfliktlinie „Arbeitsplätze und Umweltschutz“ bereits seit dem Beginn der Industrialisierung den Zugang der ArbeiterInnenbewegung zur Natur. Ab den 1980er-Jahren lässt sich jedoch hinsichtlich dieses ,klassisch gewordenen Konflikt[s]“ (Sandner 1999, S. 339) ein Bruch feststellen. Während Umweltschutz in den Jahrzehnten zuvor, vor dem Hintergrund von Vollbeschäftigung, entkoppelt von beschäftigungspolitischen Aspekten diskutiert wurde, kam dieser mit den Rezessionen 1974 und 1981 ,ins beschäftigungspolitische Gerede“ (Ritt 1998, S. 5) und wurde als Chance betrachtet. Über die so genannte „double dividend“, also den ,,positiven Beschäftigungswirkungen von Umweltschutz“ (Brandl und Hildebrandt 2002, S. 53) bildete sich eine ,eigenartige Koalition von Arbeitsmarktpolitik und Umweltpolitik“ (Ritt 1998, S. 5), die sowohl von den Sozialpartnern als auch von Umweltorganisationen forciert wurde (vgl. ebd.). Obwohl diese Neuinterpretation des Spannungsverhältnisses „Arbeitsplätze und Umweltschutz“ verstärkt Umweltthemen in die gewerkschaftlichen Debatten brachte, besteht dadurch jedoch auch die Gefahr, dass Umweltpolitik auf ihre Beschäftigungswirksamkeit reduziert wird (vgl. Ritt 1998). Grundsätzlich haben gewerkschaftliche Kampagnen zu „Green 
Jobs“ zweifelsohne eine wichtige Brückenfunktion, da sie Umweltthemen innerhalb der Gewerkschaften positiv besetzen. Es besteht jedoch die Gefahr, dass jene Branchen, die mit Umweltzielen schwer vereinbar sind, wie etwa die Automobilindustrie oder die Stahlindustrie, außen vor bleiben. Vor allem in diesen Bereichen stehen Gewerkschaften vor der Herausforderung, grundsätzliche strukturelle Umbauszenarien zu entwerfen, die die Interessen von Beschäftigten mit Umweltzielen vereinbar machen.

Hinzu kommt, dass die aktuelle Debatte um „Green Jobs“ keineswegs neu ist, sondern vielmehr als ,Neuauflage einer bereits länger dauernden Auseinandersetzung um Umwelt und Arbeit interpretiert werden“ (Littig 2012, S. 585) kann.

Gleichzeitig wurde Umweltschutz im Allgemeinen und Klimaschutz im Besonderen vor dem Hintergrund der Krise jedoch auch als Bedrohung für Arbeitsplätze wahrgenommen. Besonders deutlich wird dies im Zusammenhang mit dem EUEmissionshandelssystem, das seitens der österreichischen Gewerkschaften in den vergangenen Jahren mehrmals heftig kritisiert wurde (vgl. GPA-djp 2008; Austropapier 2008).

Neben dem Forcieren von „grünen Arbeitsplätzen“ als Beschäftigungsmotor in einzelnen Branchen, wie etwa der Bauindustrie, hinterfragen Gewerkschaften jedoch das tatsächliche Potential und vor allem die Qualität der Arbeitsplätze, die in diesem Bereich entstehen (vgl. AK Österreich 2012).

Die gewerkschaftliche Herangehensweise an „Green Jobs“ ist eng mit den Sektoren, die die jeweiligen Gewerkschaften organisieren, verknüpft. Konkret lassen sich hierbei zwei unterschiedlich ausgeprägte Schwerpunkte feststellen: Einerseits die Strategie der GPA-djp und der Gewerkschaft vida, Arbeitsplätze im Sozial- und Gesundheitsbereich als „Green Jobs“ zu definieren, und andererseits die Kampagne der Gewerkschaft Bau-Holz „Umwelt + Bauen“ zur thermischen Sanierung. Arbeitsplätze im Gesundheits- und Sozialbereich gelten per Definition nicht als „Green Jobs“ (vgl. Statistik Austria 2008). Dennoch forcierten die GPA-djp und die Gewerkschaft vida neben klassischen „Green Jobs“ auch Arbeitsplätze im Bereich der sozialen Dienstleistungen und knüpfen damit an ihre bisherige Kampagne für eine „Sozialmilliarde“ zum Ausbau der sozialen Dienstleistungen an. Vor dem Hintergrund der Wirtschaftskrise fordert die GPA-djp einen „Strukturwandel“ (GPA-djp Presseaussendung, 10. November 2009), der vor allem die Bereiche ,Soziale Dienste oder Ökologie“ (ebd.) fördert und ,einen entscheidenden Impuls für eine dauerhafte Konjunkturbelebung geben“ (ebd.) soll. In eine ähnliche Richtung argumentiert die Gewerkschaft vida, die vor dem Hintergrund der Wirtschaftskrise 2008/2009 auf das Beschäftigungspotential im Sozialbereich hinweist und von „Jobs mit Zukunft“ (vida 2009, S. 5) spricht.

Die bereits erwähnte Kampagne „Umwelt + Bauen“ wurde 2010 gestartet und hat das Ziel die thermische Sanierung zu forcieren (vgl. Umwelt + Bauen 2011). Obwohl zu Beginn der Kampagne das wesentliche Motiv für die GBH ,die Schaffung von Arbeitsplätzen war“ (Interview 04), räumt ein Interviewpartner ein, dass es ausgehend von einem Organisationsentwicklungsprozess auch weitreichendere Überlegungen gibt, um die GBH als Umweltgewerkschaft zu positionieren, da ,im Umweltthema für uns die Zukunft und auch die Zukunft unserer Arbeitsplätze liegt““ (Interview 04). 
Eine Verknüpfung von sozial-, arbeitsrechtlich- und umweltrelevanten Fragen in Bezug auf Arbeitsplätze im Verkehrsbereich zeigt die Gewerkschaft vida auf (vgl. vida 2009). Hintergrund dabei ist, dass sich das Güterverkehrsgewerbe in den letzten Jahren massiv zu Lasten der Beschäftigten verändert hat. „Der Faktor Arbeit ist der weitaus wichtigste Kostenfaktor im Güterverkehr, insbesondere aber im Straßengüterverkehr! Er kann bis zur Hälfte der Transportkosten (und in Spezialfällen sogar noch weit mehr) ausmachen“ (AK Wien 2001, S. 127). Ein Interviewpartner nennt diese Verbilligung von Transport auf Kosten der Beschäftigten in diesem Bereich als eine „Grundvoraussetzung für die Globalisierung, wie wir sie kennen“ (Interview 03). Zusammenfassend lässt sich sagen, dass Green Jobs seitens der untersuchten Gewerkschaften zwar forciert, tendenziell jedoch auch kritisch hinsichtlich der Quantität, vor allem aber der Qualität betrachtet werden (vgl. AK Österreich 2012).

\section{Neue Wohlstandsmodelle als Ausgangspunkt für gewerkschaftliche Umweltpolitik}

Wie eingangs skizziert gelingt es Gewerkschaften kaum eine eigenständige Klimapolitik zu formulieren. Vielmehr wird diese entlang konkreter Problemfelder aus der gewerkschaftlichen Praxis und oftmals im Bündnis mit Arbeitgebern entworfen. Das „Verhältnis zwischen Wachstum, Arbeitsplatzerhalt und Umverteilung“ (Walk/ Brunnengräber 2000, S. 70) stellt in diesem Zusammenhang das zentrale Konfliktfeld dar. Die diesbezügliche gewerkschaftliche Praxis ist von Widersprüchen geprägt, eröffnet jedoch auch das Feld für eine neue Diskussion um eine sozial-ökologische Transition (vgl. Fischer-Kowalski und Rotmans 2009) und die daran anknüpfende Frage nach neuen Wohlstandsmodellen. Angesichts der anhaltenden ,multiplen Krise“ (Brand 2009) und der Zunahme von (globalen) Verteilungskonflikten wird deutlich, dass Gewerkschaften verstärkt nach neuen Wegen und Modellen suchen.

Die Beantwortung der Frage eines Interviewpartners: „Wenn wir nicht mehr wachsen, wie verteilen wir dann um? Und welche Kräfte können das dann bewerkstelligen?" (Interview 01) wird für die Gewerkschaftsbewegung sowohl aus sozialen als auch aus ökologischen Gesichtspunkten in nächster Zukunft von zentraler Bedeutung sein. Letztendlich verdeutlicht die aktuelle krisenhafte Entwicklung der globalen Ökonomie, dass ,ein gutes Leben für alle“ ein neues Entwicklungsmodell erfordert, das Fragen der Verteilungsgerechtigkeit aus sozialen und ökologischen Gesichtspunkten beantworten kann.

Zusammenfassend zeigt sich, dass die österreichischen Gewerkschaften durchaus zentrale ökologische Herausforderungen aufnehmen und thematisieren. Auch wenn diese oftmals noch vage formuliert bzw. lediglich auf programmatischer Ebene vorhanden sind, drückt dies zweifelsohne eine Bewusstseinsänderung in Bezug auf ökologische Fragen innerhalb der Gewerkschaftsbewegung aus. Hinzu kommt, dass mit Projekten wie „Umwelt + Bauen“ bereits erste erfolgreiche Erfahrungen gemacht und die Konkretisierung von Programmen erfolgt ist.

Wichtige Anknüpfungspunkte für weitere Aktivitäten stellen einerseits die Debatten um Nachhaltigkeit dar, die ökologische und soziale Fragestellungen verknüpften 
und gesellschaftlichen Wandel vor einem breiteren Kontext diskutieren (vgl. u. a. Littig 1998), aber auch die konkreten Erfahrungen von Gewerkschaften vor dem Hintergrund der globalisierungskritischen Bewegung. Auch aktuelle internationale Allianzen wie „Climate Justice“ lassen erste Ansätze einer neuen Strategie erkennen. Mit dem Ansatz der „Environmental Labour Studies“ gibt es zudem auch eine erste Sammlung und Systematisierung von konkreten Erfahrungen von Gewerkschaften im Umgang mit ökologischen Fragen (vgl. Räthzel und Uzzell 2013). In den letzten Jahren wurden vor allem auf internationaler gewerkschaftlicher Ebene zahlreiche Programme und Strategien erarbeitet, um sozial-ökologische Fragestellungen verstärkt in die gewerkschaftlichen Debatten zu tragen (vgl. u. a. ITUC 2008; ITF 2010). Insbesondere das Konzept von ,just transition“, das seit 2009 vom Internationalen Gewerkschaftsbund (ITUC) propagiert wird, ist in diesem Zusammenhang von zentraler Bedeutung, da es neben der Vereinbarkeit von Klimazielen und den Interessen von Beschäftigten auch die Forderung von öffentlichen Investitionen und einer längerfristig geplanten Industriepolitik zum Inhalt hat und ein alternatives Entwicklungsmodell abseits von neoliberalen und marktbasierten Lösungsansätzen einfordert (vgl. Snell und Fairbrother 2013, S. 149).

Die Implementierung solcher Ansätze in die konkrete Politikgestaltung auf nationaler Ebene stellt jedoch zweifelsohne, nicht zuletzt vor dem Hintergrund von Standort- und Wettbewerbsfragen, eine große Herausforderung dar.

Open access funding provided by University of Vienna.

Open Access Dieser Artikel wird unter der Creative Commons Namensnennung 4.0 International Lizenz (http://creativecommons.org/licenses/by/4.0/deed.de) veröffentlicht, welche die Nutzung, Vervielfältigung, Bearbeitung, Verbreitung und Wiedergabe in jeglichem Medium und Format erlaubt, sofern Sie den/die ursprünglichen Autor(en) und die Quelle ordnungsgemäß nennen, einen Link zur Creative Commons Lizenz beifügen und angeben, ob Änderungen vorgenommen wurden.

\section{Literatur}

Adler, Victor. 1924. Aufsätze, Reden und Briefe. Drittes Heft: Adler als Sozialhygieniker. Wien: Wiener Volksbuchhandlung.

AK Oberösterreich. 2010. Fakten zur sozialen Lage. Linz: AK OÖ.

AK Österreich. 2012. Green Jobs. Arbeitsbedingungen und Beschäftigungspotenziale. Informationen zur Umweltpolitik 186. Wien: AK.

AK Wien. 2001. Verkehr und Infrastruktur 14. Transportpreise und Transportkosten der verschiedenen Verkehrsträger im Güterverkehr. Wien: AK Wien.

Altvater, Elmar. 2009. Das Ende des Kapitalismus, wie wir ihn kennen. Eine radikale Kapitalismuskritik. Münster: Westfälisches Dampfboot.

Andersen, Arne. 1990. Arbeiterbewegung, Industrie und Umwelt im 19. Jahrhundert. In Ökologie, technischer Wandel und Arbeiterbewegung, Hrsg. Helmut Konrad, Arne Andersen, 69-87. Wien: Europaverlag.

APA. 1976. APA-Meldung, 17. Mai 1976. http://www.historisch.apa.at/cms/apa-historisch/meldung.html? dossierID=AHD_19781105_AHD0001\&deskriptor=DO_ZW_1\&meldungsID=17454/346679/1\& source=dossier_meldungen.html. Zugegriffen: 27. Juli 2016.

APA. 1978. APA-Meldung, 6. November 1978. http://www.historisch.apa.at/cms/apa-historisch/meldung. html?meldungsID=17454/604676/31\&source=suche.html. Zugegriffen: 25. Okt. 2015.

APA. 1984. APA-Meldung, 17. Dezember 1984. http://www.historisch.apa.at/cms/apa-historisch/meldung. html?dossierID=AHD_19841208_AHD0001\&deskriptor=DO_HA_2\&meldungsID=17454/1288258/ $1 \&$ source=dossier_meldungen.html. Zugegriffen: 25. Oktober 2015. 
APA. 1983. APA-Meldung, 10. August 1983. http://www.historisch.apa.at/cms/apa-historisch/meldung. html?meldungsID=17454/1129725/6\&source=suche.html. Zugegriffen: 25. Okt. 2015.

APA. 1984. APA-Meldung, 19. Jänner 1984. http://www.historisch.apa.at/cms/apahistorisch/meldung. html?dossierID=AHD_19841208_AHD0001\&deskriptor=DO_HA_1\&meldungsID=17454/1179014/ 1\&source=dossier_meldungen.html. Zugegriffen: 12. September 2011.

APA. 1984. APA-Meldung, 13. Dezember 1984. http://www.historisch.apa.at/cms/apa-historisch/meldung. html?dossierID=AHD_19841208_AHD0001\&deskriptor=DO_HA_2\&meldungsID=17454/1287021/ $1 \&$ source=dossier_meldungen.html. Zugegriffen: 25. Oktober 2015.

APA. 1985. APA-Meldung, 31. Oktober 1985. http://www.historisch.apa.at/cms/apa-historisch/meldung. html?meldungsID=17454/1380849/5\&source=suche.html. Zugegriffen: 25. Oktober 2015.

AUGE/UG. 2009. Klima in der Krise. Herausforderung Klimawandel aus alternativer Gewerkschaftssicht. Wien: AUGE/UG.

Austropapier. 2008. Gemeinsame Position der Sozialpartner der Papierindustrie zur Gefahr des „Carbon Leakage“. http://www.austropapier.at/fileadmin/Austropapier/Dokumente/Forderungen_zum_EUEmissionshandel.pdf. Zugegriffen: 25. Okt. 2015.

Baschnegger, Hanno. 1985. Die Kälte des Dezember. Der Illustrierte Pressespiegel zu Hainburg. Wien: Eigenverlag.

Blau, Evelyn, Norbert Weiß, und Antonia Wenisch. 1997. Die Reparaturgesellschaft. Das Ende der Wegwerfkultur. Wien: ÖGB-Verlag.

Blau, Evelyn. 1998. Ökologische Aspekte der Globalisierung. In Wieviel Globalisierung verträgt unser Land?, Hrsg. Gewerkschaft der Privatangestellten. Wien: ÖGB-Verlag.

Blau, Paul. 1999. Das Erbe verschleudert, die Zukunft verspielt. Wien: Kremayr \& Scheriau.

Blau, Paul, und Michael Häupl. 1980. SPÖ und Ökologie: Mehr Rot ins Grün, mehr Grün ins Rot. In Roter Anstoß, Hrsg. Josef Hindels, Peter Pelinka, 161-176. Wien München: Jugend und Volk.

Bramwell, Anna. 1989. Ecology in the 20th Century: a history. New Haven: Yale Univ. Press.

Brand, Ulrich. 2008. „Umwelt“ in der neoliberal-imperialen Politik. Sozial-ökologische Perspektiven demokratischer Gesellschaftspolitik. Widerspruch 4:139-148.

Brand, Ulrich. 2009. Die Multiple Krise. Dynamik und Zusammenhang der Krisendimension, Anforderungen an politische Institutionen und Chancen progressiver Politik. Berlin: Böll-Stiftung.

Brand, Ulrich, und Markus Wissen. 2011. Sozial-ökologische Krise und imperiale Lebensweise. Zu Krise und Kontinuität kapitalistischer Naturverhältnisse. In VielfachKrise im finanzmarktdominierten Kapitalismus, Hrsg. Alex Demirovic, Julia Dück, Florian Becker, und Pauline Bader, 79-94. Hamburg: VSA.

Brand, Ulrich, und Adam Pawloff. 2014. Selectivities at work: climate concerns in the midst of corporatist interests. The case of Austria. Journal of Environmental Protection 5(2014):780-795.

Brandl, Sebastian, und Eckart Hildebrandt. 2002. Arbeit und Ökologie. In Sozial-ökologische Forschung. Ergebnisse der Sondierungsprojekte aus dem BMBF-Förderschwerpunkt, Hrsg. Ingrid Balzer, Monika Wächter, 517-538. München: ökom.

Brunnengräber, Achim, Kristina Dietz, Bernd Hirschl, Heike Walk, und Melanie Weber. 2008. Das Klima neu denken. Eine sozial-ökologische Perspektive auf die lokale, nationale und internationale Klimapolitik. Münster: Westfälisches Dampfboot.

Delapina, Thomas. 2011. Wachstum. Skriptenreihe des VÖGB. Wien: Verlag des VÖGB.

Dörre, Klaus. 2007. Einführung. Gewerkschaften und die kapitalistische Landnahme: Niedergang oder strategische Wahl? In Und jetzt? Politik, Protest und Propaganda, Hrsg. Heinrich Geiselberger, 53-78. Frankfurt am Main: Suhrkamp.

Dworczak, Hermann. 1985. Linke und Gewerkschafter gegen Hainburg. In Die Schlacht der Bäume. Hainburg 1984, Hrsg. Günther Nenning, Andreas Huber, 106-109. Wien: Hannibal.

Fischer-Kowalski, Marina, und Jan Rotmans. 2009. Conceptualizing, observing and influencing socialecological transitions. Ecology \& Society 14(2):3.

Felli, Roman. 2014. An alternative socio-ecological strategy? International trade unions' engagement with climate change. Review of International Political Economy 21(3):372-398.

Gärtner, Edgar. 1990. Arbeiterbewegung und Ökologie. In Ökologie, technischer Wandel und Arbeiterbewegung, Hrsg. Helmut Konrad, Arne Andersen, 33-44. Wien: Europaverlag.

GBH. 2014. Leitantrag und Anträge, 19. Gewerkschaftstag. Wien.

Glatz, Harald. 1984. Gewerkschaften und Umweltpolitik. In Umdenken. Analysen grüner Politik in Österreich, Hrsg. Werner Katzmann, 125-137. Wien: Junius.

GPA-djp. 2008. Weniger Emissionen und mehr Arbeitsplätze in Europa. Europäischer Aktionstag für Arbeitsplätze und Umweltschutz. http://www.gpa-djp.at/servlet/ContentServer?pagename=GPA/Page/ Index\&n=GPA_11.1.4.a\&cid=1227275597261. Zugegriffen: 25. Okt. 2015. 
GPA-djp. 2014. Presseaussendung, 10. November 2009. GPA-djp-Katzian zu Pröll: An weiterem Konjunkturpaket führt kein Weg vorbei. http://www.gpa-djp.at/servlet/ContentServer?pagename=GPA/Page/ Index\&n=GPA_11.1.5.a\&cid=1257346723357. Zugegriffen: 25. Oktober 2015.

GPA-djp. 2015. Anträge zum Bundesforum 2015. Wien: GPA-djp.

Gregory, Denis, Eckart Hildebrandt, Kees Le Blansch, und Borge Lorentzen. 1999. Industrial relations and protection of the environment: research findings from a new policy field. European Journal of Industrial Relations 5(2):139-162.

Hainburger-Au. 2011. http://www.hainburger-au.at/default.php?page=zeittafel\&id=unter. Zugegriffen: 17. September 2011.

Harmsen, Ger. 1990. Sozialismus, Natur, Umwelt. In Ökologie, technischer Wandel und Arbeiterbewegung, Hrsg. Helmut Konrad, Arne Andersen, 88-104. Wien: Europaverlag.

Hermand, Jost. 1991. Grüne Utopien in Deutschland. Zur Geschichte des ökologischen Bewußtseins. Frankfurt am Main: Fischer.

Hermand, Jost. 1993. 2. Köln: Böhlau.

Hesoun, Josef, und Herbert Pötschacher. 1985. Schwarz-Weißbuch. Dokumentation Hainburg. Wien: Eigenverlag.

ILO. 2002. Sustainable development of the global economy: a trade union perspective. http://ilo.org/public/ english/protection/ses/download/docs/sustain.pdf. Zugegriffen: 17. November 2015.

ITF. 2010. Verkehrsbeschäftigte und Klimawandel. Für eine nachhaltige kohlenstoffarme Mobilität. London: ITF.

ITUC. 2008. Trade unions and climate change: Equity, justice \& solidarity in the fight against climate change. http://www.ituc-csi.org/IMG/pdf/No_45_-_TradeUnions_ClimateChange_COP14.pdf. Zugegriffen: 17. November 2015.

Lackner, Helmut. 1990. Die Mitarbeit der Gewerkschaften bei der Produktivitätssteigerung nach dem 2. Weltkrieg in Österreich. In Ökologie, technischer Wandel und Arbeiterbewegung, Hrsg. Helmut Konrad, Arne Andersen, 207-216. Wien: Europaverlag.

Lebensministerium. 2012. Die österreichische Strategie zur Anpassung an den Klimawandel. Wien: Lebensministerium.

Leisch, Wilfried. 2009. GewerkschafterInnen gegen Atomenergie und Krieg. In Kein Kernkraftwerk in Zwentendorf. 30 Jahre danach, Hrsg. Heimo Halbrainer, Elke Murlasits, und Sigrid Schönfelder, 104-111. Weitra: Bibliothek der Provinz.

Linse, Ulrich. 1993. Das Proletariat - Komplize der kapitalistischen Naturausbeutung? In Mit den Bäumen sterben die Menschen. Zur Kulturgeschichte der Ökologie, Hrsg. Jost Hermand, 119-148. Köln: Böhlau.

Littig, Beate. 1998. Ökologie und soziale Krise. Wie zukunftsfähig ist die Nachhaltigkeit. Wien: Edition Volkshochschule.

Littig, Beate. 2012. Von Rio 1992 zu „Rio+20“. Arbeit im Kontext der aktuellen Nachhaltigkeitsdiskussion. WSI-Mitteilungen 8:581-588.

Littig, Beate, und Erich Grießler. 2001. Umwelt und Arbeit. Integrierter Umweltschutz - innerbetriebliche Veränderungen und Partizipation. In Informationen zur Umweltpolitik 147. Wien: Bundeskammer für Arbeiter und Angestellte.

Natter, Bernhard. 1987. Die „Bürger“ versus die „Mächtigen“ - Populistischer Protest an den Beispielen Zwentendorf und Hainburg. In Populismus in Österreich, Hrsg. Anton Pelinka, 151-171. Wien: Junius.

Nenning, Günther, und Andreas Huber. 1985. Die Schlacht der Bäume. Hainburg 1984. Wien: Hannibal.

ÖGB. 2013. Leitantrag beschlossen am 18. Bundeskongress. Wien.

Österreichische Sozialpartner. 2008. Positionspapier der österreichischen Sozialpartner. Bausteine einer langfristig orientierten Klimapolitik. Wien.

ÖGB Flugblatt. 2011. Nein zu Sparmaßnahmen. Für ein soziales Europa. Für gerechte Bezahlung und Jobs.

Passadakis, Alexis, und Tadzio Müller. 2009. Klimaschutz durch Wirtschaftswachstum. Blätter für deutsche und internationale Politik 4(20.9):33-35.

PRO-GE. 2013. Innovativ Stark Sozial. Arbeitsprogramm. Wien: PRO-GE.

Räthzel, Nora, und David Uzzell. 2013. Trade Unions in the Green Economy. Working for the environment. London New York: Routledge.

Ritt, Thomas. 1998. Umwelt und Arbeit. In Umwelt und Arbeit - Bestandsaufnahme und Perspektiven. Umwelt und Arbeit IV, Hrsg. Thomas Ritt. Wien: Bundeskammer für Angestellte und Arbeiter Wien.

Rosemberg, Anabella. 2010. Building a Just Transition: The linkages between climate change and employment. International Journal of Labour Research 2(2):125-156. 
Sandner, Günther. 1999. Die Natur und ihr Gegenteil. Politische Diskurse der sozialdemokratischen Kulturbewegung bis 1933/34. Wien: Lang.

Snell, Darryn, und Peter Fairbrother. 2013. Just transition and labour environmentalism in Australia. In Trade Unions in the Green Economy. Working for the environment, Hrsg. Nora Räthzel, David Uzzell, 146-161. London New York: Routledge.

Scheffran, Jürgen. 2004. Energiekonflikte und Klimakatastrophe. Die neue Bedrohung. PROKLA 135:173198.

Schramm, Engelbert. 1990. Arbeiterbewegung und industrielle Umweltprobleme. Wahrnehmung und Theoriediskussion in der zweiten Hälfte des 19. Jahrhunderts (bis 1918). In Ökologie, technischer Wandel und Arbeiterbewegung, Hrsg. Helmut Konrad, Arne Andersen, 1-32. Wien: Europaverlag.

Statistik Austria. 2008. Umweltorientierte Produktion und Dienstleistungen - EGSS. Wien: Statistik Austria.

Tálos, Emmerich. 1997. Sozialpartnerschaftliche Kooperation - Konzertierung - politische Regulierung. In Handbuch des politischen Systems Österreichs. Die zweite Republik, Hrsg. Herbert Dachs, 432-452. Wien: Manz.

Umwelt + Bauen. 2011. Broschüre der Initiative Umwelt + Bauen. Wien: Umwelt + Bauen. UNEP. 1992. Agenda 21. Geneva: UNEP.

vida. 2009. Zeitschrift für Mitglieder der Gewerkschaft vida. März/April 2009. Wien: vida.

vida. 2010. Grundsatzprogramm beschlossen am 2. Gewerkschaftstag. Wien: vida.

Walk, Heike, und Achim Brunnengräber. 2000. Die Globalisierungswächter. NGOs und ihre transnationalen Netze im Konfliktfeld Klima. Westfälisches Dampfboot: Münster.

Wege aus der Krise. 2014. Weniger arbeiten, mehr leben. Wien: Wege aus der Krise.

Winkler-Rieder, Waltraud. 1997. Energiepolitik. In Handbuch des politischen Systems Österreichs.Die zweite Republik, Hrsg. Herbert Dachs, 619-627. Wien: Manz.

Kathrin Niedermoser ist Sozialwissenschafterin und arbeitet am Institut für Politikwissenschaft an der Universität Wien. 